ACCEPTED MANUSCRIPT

\title{
Grain selective Cu oxidation and anomalous shift of graphene 2D Raman peak in the graphene-Cu system
}

To cite this article before publication: Javier Bartolomé et al 2018 2D Mater. in press https://doi.org/10.1088/2053-1583/aaef48

\section{Manuscript version: Accepted Manuscript}

Accepted Manuscript is "the version of the article accepted for publication including all changes made as a result of the peer review process, and which may also include the addition to the article by IOP Publishing of a header, an article ID, a cover sheet and/or an 'Accepted Manuscript' watermark, but excluding any other editing, typesetting or other changes made by IOP Publishing and/or its licensors"

This Accepted Manuscript is @ 2018 IOP Publishing Ltd.

During the embargo period (the 12 month period from the publication of the Version of Record of this article), the Accepted Manuscript is fully protected by copyright and cannot be reused or reposted elsewhere.

As the Version of Record of this article is going to be / has been published on a subscription basis, this Accepted Manuscript is available for reuse under a CC BY-NC-ND 3.0 licence after the 12 month embargo period.

After the embargo period, everyone is permitted to use copy and redistribute this article for non-commercial purposes only, provided that they adhere to all the terms of the licence https://creativecommons.org/licences/by-nc-nd/3.0

Although reasonable endeavours have been taken to obtain all necessary permissions from third parties to include their copyrighted content within this article, their full citation and copyright line may not be present in this Accepted Manuscript version. Before using any content from this article, please refer to the Version of Record on IOPscience once published for full citation and copyright details, as permissions will likely be required. All third party content is fully copyright protected, unless specifically stated otherwise in the figure caption in the Version of Record.

View the article online for updates and enhancements. 


\title{
Grain selective $\mathrm{Cu}$ oxidation and anomalous shift of graphene 2D Raman peak in the graphene-Cu
} system

\author{
Javier Bartolomé ${ }^{1,2, *}$, Leo Álvarez-Fraga ${ }^{1}$, Montserrat X. Aguilar-Pujol ${ }^{1}$, Sandra \\ Cortijo $^{1}$, Ana Cremades ${ }^{2}$, Carlos Prieto ${ }^{1}$, and Alicia de Andrés ${ }^{1}$ \\ ${ }^{1}$ Instituto de Ciencia de Materiales de Madrid, Consejo Superior de Investigaciones Científicas, C/Sor \\ Juana Inés de la Cruz 3, Cantoblanco, 28049 Madrid, Spain \\ ${ }^{2}$ Departamento de Física de Materiales, Universidad Complutense de Madrid, Plaza Ciencias 1, 28040 \\ Madrid, Spain \\ * Author to whom any correspondence should be addressed \\ E-mail: j.bartolome@fis.ucm.es
}

Received xxxxxx

Accepted for publication $\mathrm{xxxxxx}$

Published xxxxxx

\begin{abstract}
Understanding the interaction between graphene and its supporting substrate is of paramount importance for the development of graphene based applications. In this work the interplay of the technologically relevant graphene-Cu system is investigated in detail as a function of substrate grain orientation in Cu polycrystalline foils. While (100) and (111) Cu grains show the well-known graphene-enhanced oxidation, (110) grains present a superior oxidation resistance compared to uncovered $\mathrm{Cu}$ and an anomalous shift of its graphene 2D Raman band which cannot be explained by the known effects of strain and doping. These results are interpreted in terms of a weak graphene-Cu coupling at the (110) grains, and show that graphene can actually be used as anticorrosion coating, contrary to previously reported. The anomalous shift is suggested to be the result of an enhanced outer Raman scattering process which surpasses the usually dominant inner process. Since Raman spectroscopy is widely used as first and main characterization tool of graphene, the existence of an anomalous shift on its 2D band not only challenges the current theory of Raman scattering in graphene, but also has profound implications from an experimental point of view.
\end{abstract}

Keywords: graphene, Raman spectroscopy, copper oxidation, strain, graphene-substrate interaction

\section{Introduction}

Chemical vapor deposition (CVD) on polycrystalline $\mathrm{Cu}$ foils is one of the most widely used methods for obtaining low cost, large area graphene. Thus, great efforts have been invested during the last years in the study of the interaction between graphene and its single or polycrystalline $\mathrm{Cu}$ substrates. The effect of $\mathrm{Cu}$ crystalline orientation on the nucleation [1, 2], growth [3, 4] and physical properties [5] of graphene has been investigated in detail in the past, whereas the influence of graphene on the oxidation mechanisms of $\mathrm{Cu}$ has drawn an increasing attention due to its prospective applications as protective layer [6-10]. This last topic is currently the focus of an intense research in the field of 2D materials, not only for their use as anticorrosion coatings, but also because many of these materials show tendency to degrade under ambient conditions [11-13]. Thus, searching 
for suitable protective materials which could be integrated in the architecture of 2D devices is on main interest for this emergent industry. In the case of graphene coated copper, previous works showed that, while graphene is able to significantly slow down the oxidation of $\mathrm{Cu}$ in the short term, it cannot totally stop the process, and may actually produce the opposite effect in the long term as graphene overrides the protective behavior of the native oxide layer $[7,8,10]$. On the other hand, the growth of an intercalated oxide layer has turned out to be of interest on its own as it has been demonstrated to produce an interference enhancement of the Raman signal $[14,15]$ as well as to aid the detachment of graphene from the $\mathrm{Cu}$ substrate $[16,17]$. However, despite its ubiquity and interest, little attention has been paid to the interaction between graphene and the intercalated copper oxide layer. The effect of copper oxide on the Raman spectra of graphene grown on polycrystalline $\mathrm{Cu}$ foils was studied by Yin et al. [14], but they did not distinguish between the different grain orientations of the foil. It is well known that the oxidation rate of bare $\mathrm{Cu}$ depends on the crystal orientation of the exposed surface. The oxidation kinetics of different $\mathrm{Cu}$ surfaces has been subject of many studies over the last decades [18-23]; however, the oxidation process itself is influenced by a number of factors, such as the morphology and surface defects of the sample [22, 24, 25], its thermal history [10] or the oxidation temperature $[18,25]$, which has led to apparent contradictory results. It is thus expected that both, the oxidation process of graphene covered $\mathrm{Cu}$, and the interaction between graphene and the intercalated oxide layer, would depend on the exposed $\mathrm{Cu}$ surface. To take full advantage of the new capabilities provided by the intercalated oxide layer and the possibility of tuning the foil texture [26], it is necessary to understand how the different crystallographic orientation of its grains affects the oxidation process in the graphene- $\mathrm{Cu}$ system. Besides, since the use of polycrystalline $\mathrm{Cu}$ foils has been posed as one of the most suitable options for mass production of graphene, it is important to know how graphene properties are modified by the $\mathrm{Cu}$ substrate and the intercalated oxide layer, as a function of the grain orientation.

Raman spectroscopy is a widely used characterization technique for the study of graphene, and other 2D materials, as it is nondestructive, requires relatively simple setups and it is capable of providing information on the amount of defects, number of layers, electronic structure and mechanical state of the samples. Because of this, it is also used in the industry as the main tool to assess the properties and quality of the produced graphene. However, Raman spectroscopy has as main disadvantage its more complex data interpretation. Significant efforts have been devoted to the study of the Raman spectra on graphene and its interpretation [27, 28]. Yet, there are still some fundamental issues which are not completely understood. For instance, the commonly reported single component nature of the $2 \mathrm{D}$ peak is usually explained in terms of perfectly conical electronic and phononic band structures around the $K$ points. However, due to the trigonal warping effect both bands are distorted at increasingly large energies, leading to nonequivalent directions in the Brillouin zone (see figure S1). Therefore a multicomponent 2D band should be expected instead. Even if the scattering process is modeled considering only phonons along the high symmetry directions, it would give raise to two distinct 2D components, contrary to what is commonly observed. Interestingly, recent works have shown that the line shape of the 2D band of freestanding graphene is actually asymmetric and can be fitted to two components $[29,30]$, while it becomes symmetric on supported or electrostatically doped graphene [31,32], suggesting that the contribution from phonons along different directions cannot be neglected. Understanding the asymmetric nature of the 2D band and its symmetrisation upon doping/substrate interaction would give a deeper insight on the involved processes, which are of fundamental importance for the correct interpretation of the Raman spectra of supported graphene. Since/this phenomenon has not been studied for graphene on metallic supports, the graphene$\mathrm{Cu} / \mathrm{Cu}_{2} \mathrm{O}$ system provides an excellent platform to explore it, also as a function of the metal substrate orientation.

In this work, we investigate the orientation dependent oxidation of graphene covered $\mathrm{Cu}(\mathrm{Gr} / \mathrm{Cu})$ foils, and how their properties are affected by the orientation of the $\mathrm{Cu}$ grains and the presence of an intercalated oxide layer. To this end we determined the crystallographic orientation of different $\mathrm{Cu}$ foil grains by means of electron backscattering diffraction (EBSD) and studied their oxidation over a period of more than 8 months under ambient conditions. Conventional and cross polarized (CP) optical microscopy, as well as atomic force microscopy (AFM) and scanning electron microscopy (SEM) were used to track the changes in morphology and oxide coverage of the samples, while Raman spectroscopy was used to characterize the physical properties of graphene as well as to qualitatively compare the oxidation degree of the samples. Our study reveals important changes in the oxidation kinetics of graphene covered $\mathrm{Cu}$ compared to uncovered one, manifested in a swap of the oxidation rate relationship between the three low Miller index orientations, (100), (110) and (111), with (100) and (111) oriented grains oxidizing faster in the graphene covered $\mathrm{Cu}$, and the (110) grains showing qualitatively lower long term oxidation degrees compared to the bare $\mathrm{Cu}$. This difference is correlated to an orientation dependent modification of the surface morphology of the grains, which is produced during the growth of graphene. The oxide layer itself is found to tensile strain and dope the otherwise compressively strained and undoped graphene, with little effect of the $\mathrm{Cu}$ grain orientation. An important and unexpected result is the observation of an anomalous shift of the $G$ and 2D Raman peaks of graphene on $\mathrm{Cu}$ (110) unoxidized surfaces, which is removed upon 
oxidation. This shift, which has been commonly overlooked in the literature, is correlated with a significant lower oxidation rate and surface smoothness of (110) grains compared to (100) and (111) ones, and cannot be explained by the usual substrate strain/doping effect.

Understanding the oxidation process of $\mathrm{Cu}$ below graphene and the physical phenomena behind the anomalous shift of the 2D peak of graphene supported on $\mathrm{Cu}$ is crucial for the implementation of the CVD growth of graphene on $\mathrm{Cu}$ in the industry as well as for the correct interpretation of the Raman spectra, which are systematically used to characterize graphene.

\section{Experimental section}

\subsection{Sample fabrication}

Graphene single layers were grown on $\mathrm{Cu}$ foils by $\mathrm{CVD}$ following a procedure reported elsewhere [10]. Briefly, commercial $\mathrm{Cu}$ foils (25 $\mu$ m-thick, $99.8 \%$, Alfa Aesar) were first cleaned with acetone and acetic acid to remove any trace of surface contaminants, and then annealed at $250{ }^{\circ} \mathrm{C}$ in air to form a protective oxidized surface. The foils were then transferred into a CVD tubular furnace, where they were annealed at $1015{ }^{\circ} \mathrm{C}$ in the presence of a hydrogen flux $\left(\sim 8 \cdot 10^{-1} \mathrm{mbar}, 104 \mathrm{sccm}\right)$ for $1 \mathrm{~h}$. Afterwards the temperature was decreased to $900{ }^{\circ} \mathrm{C}$ and an additional $\mathrm{CH}_{4}$ flux of 10 sccm was introduced for 30 minutes to induce the growth of graphene. To perform a proper comparison, control samples, i.e. $\mathrm{Cu}$ foils with no graphene on top, were grown following the same procedure as for the $\mathrm{Gr} / \mathrm{Cu}$ foils, but without introducing $\mathrm{CH}_{4}$ during the $900{ }^{\circ} \mathrm{C}$ step to avoid the formation of graphene. Cooling was performed by placing the samples on the cold region of the tube for about 15 minutes before venting. The samples were stored in ambient conditions after growth and studied for more than 8 months as they oxidized.

\subsection{Characterization techniques}

The morphology of the samples was studied by means of optical microscopy, both in bright field and CP modes, as well as by SEM in a FEI Inspect microscope with accelerating voltages in the range of 5-20 V. AFM images were collected in tapping mode (Nanosensors PPP-NCH-w Si tips with cantilever resonance frequency $f_{0} \sim 270 \mathrm{kHz}$ and $k \sim 30 \mathrm{~N} \mathrm{~m}^{-1}$ ) with a commercial equipment from Nanotec. Orientation maps were obtained by means of EBSD carried out with a Bruker e-FlashHR ${ }^{+}$detector using an acceleration voltage of $20 \mathrm{kV}$, and a sample tilt angle of $70^{\circ}$. The recorded Kikuchi patters were automatically analyzed by the QUANTAX CrystAlign software in order to determine the crystalline orientation of the foils at each probed pixel. This technique has a typical probing depth of tens of nm, which has two implications: first, EBSD is not a surface technique; the overall crystalline orientation of the grains is given with respect to the sample surface and thus it is insensitive to surface reconstructions or terrace step formation at low angle surfaces. Second, even if there is an oxide layer atop, its thickness is typically of few nm [26], and thus the majority of the signal comes from the $\mathrm{Cu}$ below, allowing the identification of the crystal orientation of the underlying $\mathrm{Cu}$. Micro-Raman experiments were performed at room temperature using the $488 \mathrm{~nm}$ line of an $\mathrm{Ar}^{+}$laser with an Olympus microscope (100x objective with a numerical aperture of NA $=0.95$, which corresponds to a Raman spatial resolution of $\sim 0.8 \mu \mathrm{m}$ for $488 \mathrm{~nm}$ ) and a "super-notch-plus" filter from Kaiser. The scattered light was analyzed with a Jobin-Yvon HR-460 monochromator coupled to a Peltier cooled Synapse CCD. Reference freestanding graphene Raman spectra were recorded on CVD graphene transferred onto an $\mathrm{Al}_{2} \mathrm{O}_{3} / \mathrm{Al}$ foil. The foil was bended prior the transference of graphene in order to induce cracks on the alumina, and measurements were performed on graphene suspended over the cracks.

\section{Results and discussion}

\subsection{Grain dependent oxidation of graphene covered $\mathrm{Cu}$ foils}

After several months of air exposure both the $\mathrm{Gr} / \mathrm{Cu}$ foils and the control sample present grains with different color contrast under the optical microscope. The difference in color contrast is a direct indication of different oxidation degrees, the redder tones being related to a more advanced oxidation state, as confirmed by Raman spectroscopy. Figures 1(a) and (b) shows representative micrographs of both samples, as well as their corresponding orientation maps, where some low index grains have been identified, namely (100), (110) and (111) grains. Raman spectra recorded at the identified grains are plotted in figures 1(c) and (d), respectively.

The set of peaks at frequencies below $1000 \mathrm{~cm}^{-1}$ has been attributed to $\mathrm{Cu}_{2} \mathrm{O}$ phonon modes [10], while the two peaks located at $\sim 1580$ and $\sim 2700 \mathrm{~cm}^{-1}$ (in the case of the $\mathrm{Gr} / \mathrm{Cu}$ foil) are the characteristic $\mathrm{G}$ and $2 \mathrm{D}$ peaks of graphene, respectively. The control sample also presents two very broad peaks in the spectral region of $1350-1600 \mathrm{~cm}^{-1}$, which correspond to carbonaceous species deposited during the EBSD measurements performed after it was already oxidized. The intensity of the $\mathrm{Cu}_{2} \mathrm{O}$ peaks is related to the oxide thickness on the probed area, and therefore its most intense peak, centered at $\sim 640 \mathrm{~cm}^{-1}$, can be used to qualitatively compare the oxidation degree of different grains [7, 10]. It is clear from the spectra, as well as from the different reddish tone of the samples, that the $\mathrm{Gr} / \mathrm{Cu}$ foil is more oxidized with respect to the control sample, as expected after a long oxidation period [7, 8, 10]. Comparison of grains with different orientations shows that in the control sample the (110) grains are more oxidized, followed by the (111) grains 
and then the (100) grains, although these variations are relatively small $\left(r_{\text {Cu foil }}^{(100)}<r_{\text {Cu foil }}^{(111)}<\boldsymbol{r}_{\text {Cu foil }}^{(\mathbf{1 1 0})}\right.$, where $r$ stands for oxidation rate, figure $1(\mathrm{c}))$. In general (100) surfaces are reported to have the fastest oxidation rates at room temperature, contrary to our own results, while no agreement is found concerning (110) and (111) surfaces [33, 34]. However, theoretical calculations, have shown that structural disorder can swap the oxidation rate relationship of these surfaces, as atomic defects (and/or temperature) increase the oxidation rate of (110) and (111) surfaces with respect to (100) ones, which are almost insensitive to this effect [25]. Surface defects are expected in large amounts in the studied foils due to the presence of extrusion lines (surface steps) and relatively fast cooling after sample growth (point defects), thus explaining our observations. This result highlights the importance of comparing the oxidation rates of different crystallographic orientations between samples treated under similar conditions.
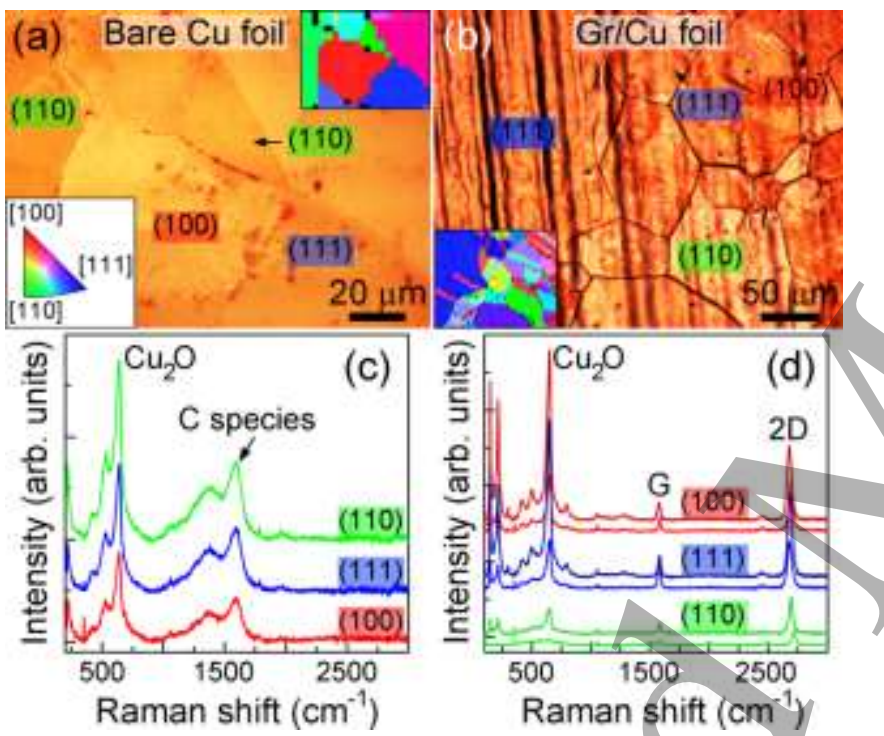

Figure 1. (a) and (b) Optical micrographs of the control sample and the $\mathrm{Gr} / \mathrm{Cu}$ foil after several months of air exposure. Some low index grains are indicated. Insets show the corresponding orientation maps as determined by EBSD. The orientation color code is displayed at the bottom left. (c) and (d) Raman spectra acquired at different grains of the control sample and the $\mathrm{Gr} / \mathrm{Cu}$ foil, respectively. Due to the inhomogeneous distribution of the oxide layer on the $\mathrm{Gr} / \mathrm{Cu}$ foil, two spectra, representative of the highly and lowly oxidized areas, are shown in (d). The two peaks at $1350-1600$ $\mathrm{cm}^{-1}$ on (c) correspond to carbonaceous species deposited by the SEM during the EBSD measurements.

The situation is exactly the opposite for the $\mathrm{Gr} / \mathrm{Cu}$ foil, with faster oxidation rates for grains oriented parallel to the (100) and (111) surfaces, while (110) grains present large unoxidized areas, as confirmed by the absence of the $\mathrm{Cu}_{2} \mathrm{O}$
Raman peak (within the detection limit of the technique), and some small and weakly oxidized patches, $\left(\boldsymbol{r}_{G \boldsymbol{r} / \boldsymbol{C u}}^{(110)} \ll r_{G r / C u}^{(111)} \lesssim\right.$ $r_{G r / C u}^{(100)}$, figure $\left.1(\mathrm{~d})\right)$. The difference in oxidation rates is much higher in the $\mathrm{Gr} / \mathrm{Cu}$ foil than in the control sample, as evidenced from the comparison of the Raman spectra. Therefore, the presence of graphene drastically changes the oxidation kinetics of the different surfaces, swapping their oxidation rate relationship to the point of enhancing the long term oxidation of $\mathrm{Cu}(100)$ and (111) grains, while hindering the same process on (110) grains. This suggests that graphene can actually work as a protective layer under certain circumstances, contrary to the general graphene-enhanced corrosion reported previously, where the orientation of the substrate was not considered.

The inversion in the oxidation rate relationship is accompanied by a change in both the morphology and the oxidation habit of the grains. Figure 2 shows the optical, CP and SEM images of both the control sample and the $\mathrm{Gr} / \mathrm{Cu}$ foil. While the contrast in the optical images is mainly related to the presence of $\mathrm{Cu}_{2} \mathrm{O}$, the $\mathrm{CP}$ and SEM images are sensitive to both the topography and the presence of $\mathrm{Cu}_{2} \mathrm{O}$ (the latter due to the interference signal amplification effect of $\mathrm{Cu}_{2} \mathrm{O}$ [15] and its different electron yield compared to bare $\mathrm{Cu}$, respectively). As can be seen in figures $2(\mathrm{a}-\mathrm{c})$, the control sample shows a relatively homogeneous oxidation degree within each grain, despite different grains present different oxidation degrees. The $\mathrm{Gr} / \mathrm{Cu}$ foil, on the other hand, has an uneven distribution of $\mathrm{Cu}_{2} \mathrm{O}$ within each grain, with large, elongated oxide patches covering the surface of (100) and (111) grains, and small $\mathrm{Cu}_{2} \mathrm{O}$ islands scattered over the otherwise pristine (110) grains (figures $2(\mathrm{~d}-\mathrm{l})$ ). Besides, while the control sample looks relatively smooth regardless the considered grain (figures $2(\mathrm{a}-\mathrm{c})$ ), the $\mathrm{Gr} / \mathrm{Cu}$ foil clearly shows a different morphology depending on the grain orientation, with (100) and (111) grains presenting rippled surfaces, and (110) grains showing pretty smooth surfaces instead (figures $2(d-1)$ ). Similar ripples on grains with the same (100) and (111) orientation (but not on (110) grains) can be observed in the as grown $\mathrm{Gr} / \mathrm{Cu}$ samples (figure $2(\mathrm{~m})$ ), evidencing that this is a general result and that ripples are formed during the growth of graphene and not as a result of the oxidation process. AFM images show that these ripples are actually composed of a large number of small terrace steps with a typical step height of $5-30 \mathrm{~nm}$ (see figures $3(\mathrm{a})$ and (b)). This result suggests that the ripples are most probably originated by the bunching of $\mathrm{Cu}$ steps moving due to the sublimation of the foil during the thermal treatment, as reported by Wofford et al. [1] and Hayashi et al. [35]. The step bunching process is produced when the terrace step movement is stopped or slowed down by the presence of a graphene layer, as it prevents the $\mathrm{Cu}$ atoms from escaping the foil surface (see the sketch in figure 3(c)). Rippling is not limited to (100) and (111) surfaces, but it is also present in other (but not all) higher 
Miller index surfaces. All these step-bunched surfaces show similarly high oxidation degrees, suggesting that the variation in oxidation rate is somehow related to the presence of $\mathrm{Cu}$ ripples.
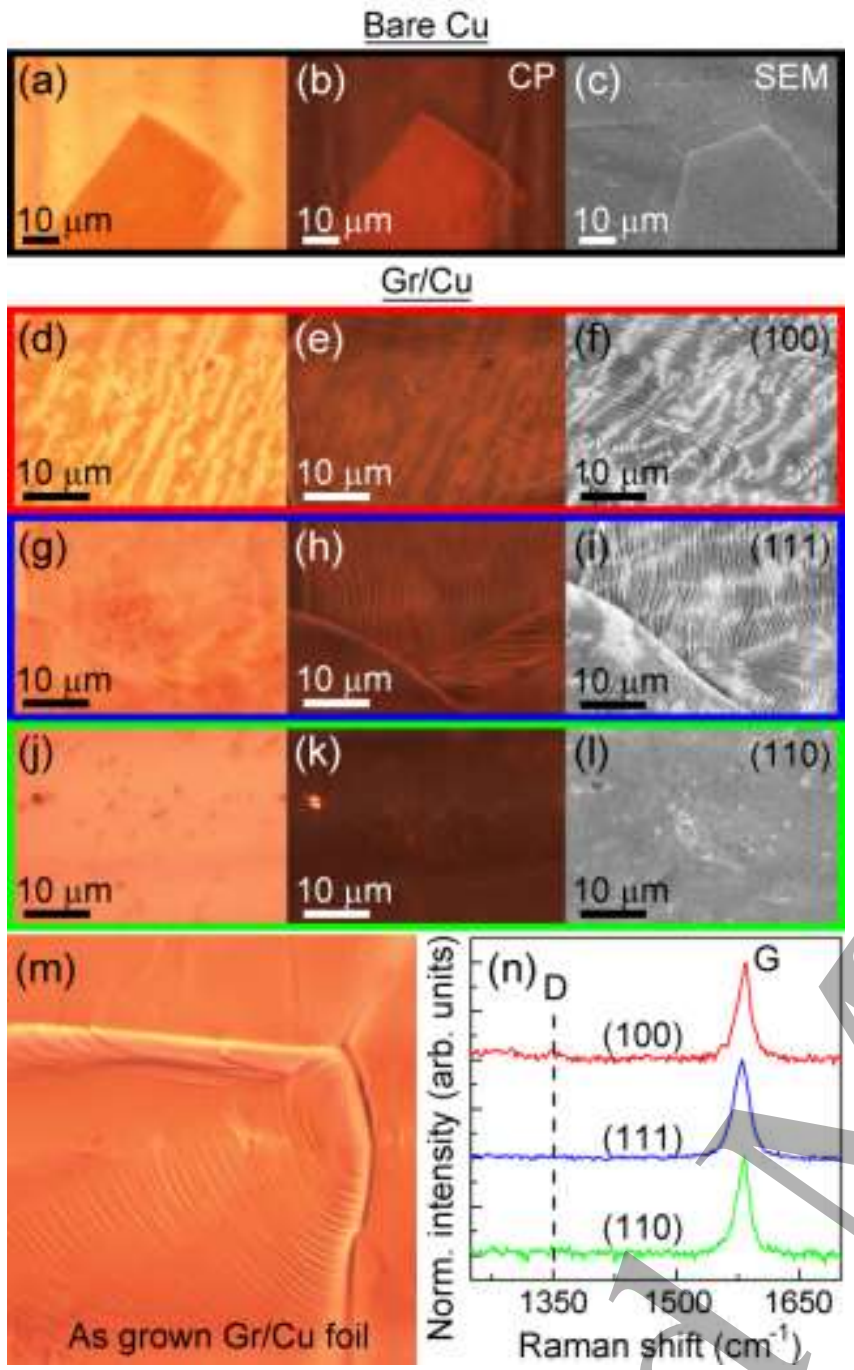

Figure 2. Optical, cross polarized (CP) and SEM images of the different $\mathrm{Cu}$ surfaces: (a)-(c) bare $\mathrm{Cu}$ foil, (d)-(f) $\mathrm{Gr} / \mathrm{Cu}$ (100) grain, (g)-(i) $\mathrm{Gr} / \mathrm{Cu}$ (111) grain and (j)-(1) $\mathrm{Gr} / \mathrm{Cu}$ (110) grain. The redder/brighter regions correspond to the $\mathrm{Cu}_{2} \mathrm{O}$ patches in the optical/cross polarized images, respectively. In the SEM images the darker regions correspond to the $\mathrm{Cu}_{2} \mathrm{O}$ patches on (100) and (111) grains, while the contrast is reversed for (110) grains. (m) Optical image of an as grown $\mathrm{Gr} / \mathrm{Cu}$ foil showing the presence of ripples in one of the grains before any oxidation has taken place. (n) Raman spectra acquired at different grains of the $\mathrm{Gr} / \mathrm{Cu}$ foil showing the absence of D peak.
Incomplete coverage of graphene or increased concentration of graphene defects as a result of the presence of ripples are two possible explanations linking both phenomena. However, Raman maps recorded at different grains confirmed that graphene totally covers the whole surface of the sample (the laser spot size is smaller than the typical size of the oxide patches). In fact, the oxide patches are aligned approximately perpendicular to the ripples, while it was demonstrated that graphene tends to align parallel to the $\mathrm{Cu}$ terrace steps in the early growth stages, i.e. when graphene has not totally covered the foil surface [35]. Besides, despite graphene defects have been reported to act as nucleation centers for the oxidation of graphene covered $\mathrm{Cu}[6,36]$, no correlation was found between the intensity of the $D$ peak (related to defects and almost undetectable in our samples) and the presence of oxidized patches (figure 2(n)), suggesting a similarly low concentration of defects in all the studied grains.

A different explanation would rely on a grain dependent coupling between graphene and its $\mathrm{Cu}$ substrate. Those grains showing a weaker mechanical coupling will present higher surface mobility for the $\mathrm{Cu}$ ions, allowing the terrace steps to keep moving despite being covered by graphene, preventing the formation of ripples. Similarly, graphene has been reported to enhance the electrochemical oxidation of $\mathrm{Cu}$ by assisting the electron transfer from the $\mathrm{Cu}$ to the upper surface of its native oxide layer $[7,8]$. During the oxidation process $\mathrm{Cu}^{+}$ cations diffuse through the oxide layer to the surface, where they react with adsorbed $\mathrm{O}$ species in a redox reaction. Nevertheless, the insulating nature of the oxide impedes those electrons left behind to reach the surface, preventing the oxidation from continuing. Graphene can contact electrically both the $\mathrm{Cu}$ and $\mathrm{Cu}_{2} \mathrm{O}$ surfaces, allowing the redox reaction to proceed. However, if graphene is electrically decoupled from the substrate this process cannot take place, effectively protecting the $\mathrm{Cu}$, as observed in the smooth (110) surfaces. Raman spectra performed on an as-grown sample showed that those grains presenting smooth surfaces have statistically higher 2D to $\mathrm{G}$ integrated ratios, which is an indication of a lower degree of graphene-substrate coupling, compared to grains with rippled surfaces (see figure S2). Similarly, Zhang et al. [2] observed a physical decoupling between graphene and $\mathrm{Cu}(110)$ surfaces after performing some low temperature thermal cycles, while Wilson et al. [37] described a very weak interaction between graphene and $\mathrm{Cu}$ (110) single crystals in order to explain an observed weak mismatch epitaxy of graphene on that surface. 

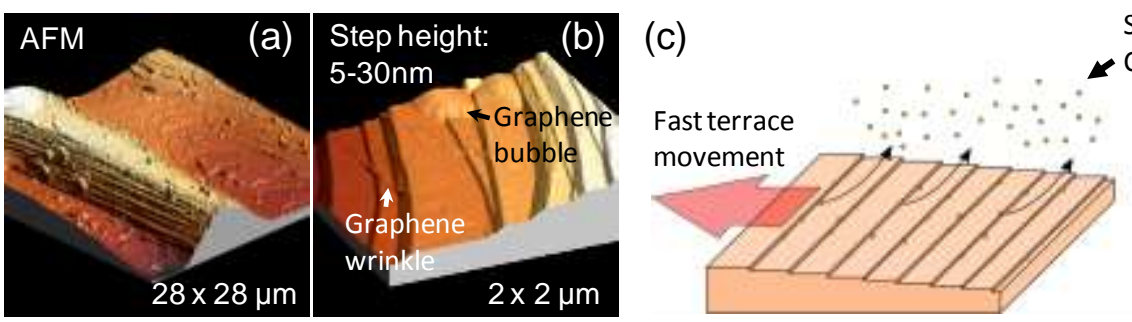

Sublimated

Figure 3. (a) and (b) AFM images of the Gr/Cu rippled surfaces. The low magnification image reveals how the observed ripples are composed of a large amount of smaller $\mathrm{Cu}$ steps, while the high magnification image shows a detail of these steps, having a step height of $5-30 \mathrm{~nm}$. (c) Sketch of the graphene driven step bunching process. Without graphene all the steps move at a constant rate as a result of the $\mathrm{Cu}$ sublimation, keeping the surfaces smooth. After graphene has nucleated it prevents the $\mathrm{Cu}$ atoms to leave the surface underneath, slowing down the movement of the steps and causing them to pile up.

\subsection{Graphene-Cu and graphene- $\mathrm{Cu}_{2} \mathrm{O}$ interaction}

Considering the clearly unavoidable oxidation of the $\mathrm{Gr} / \mathrm{Cu}$ foils as well as the totally different evolution of the grains, it seems necessary to study the effect this oxide layer has on graphene properties, taking into account the orientation of the grains. Figure 4(a) shows a representative example of the Raman spectra of the three different orientations presenting different degrees of oxidation. It is evident from the plot that the 2D peak (and the G peak to a lesser extent) shifts to lower Raman frequencies as the oxidation degree increases. This is further confirmed by the Raman maps performed at different grains as shown in figures $4(\mathrm{~b}-\mathrm{g})$. Those grains showing more reddish tones (more oxidized) have lower 2D Raman frequencies, $\omega_{2 D}$.

Figure 5(a) shows $\omega_{2 D}$ as a function of the intensity of the $\mathrm{Cu}_{2} \mathrm{O}$ peak at $640 \mathrm{~cm}^{-1}, \mathrm{I}\left(\mathrm{Cu}_{2} \mathrm{O}\right)$, for a large set of spectra recorded from (100), (110), (111) and other randomly oriented

grains (red, green, blue and grey symbols respectively). Measurements were performed at different times during a period of 8 months after the sample was grown. The observed frequency decrease is clearly related to the $\mathrm{Cu}_{2} \mathrm{O}$ thickness and has otherwise a very weak dependence on the orientation of the grains (see figure 5(a)), although (111) grains present slightly larger shifts. Black squares correspond to measurements performed on an as grown (non-oxidized) sample and depict the dispersion of $\omega_{2 D}$ for $I\left(\mathrm{Cu}_{2} \mathrm{O}\right)=0$, with an average $\omega_{2 D}$ value of $2717 \pm 7 \mathrm{~cm}^{-1}$. When all data points are plot in semi-logarithmic scale (figure 5(b)) a clear linear trend can be observed. Data can be fitted to an empirical formula $y=A-B \ln (x+C)$, with $A=2727.2 \mathrm{~cm}^{-1}, B=6.3 \mathrm{~cm}^{-}$ ${ }^{1}$ and $C=4.4$, with a saturation value of $y\left[I\left(\mathrm{Cu}_{2} \mathrm{O}\right)=0\right]=2718$ $\mathrm{cm}^{-1}$, which is very approximately the average value of $\omega_{2 D}$ for the as grown, non-oxidized $\mathrm{Gr} / \mathrm{Cu}$ foil. It is worth noting that (111) grains show some points which deviate from the observed trend, although the reason for this is still unclear.
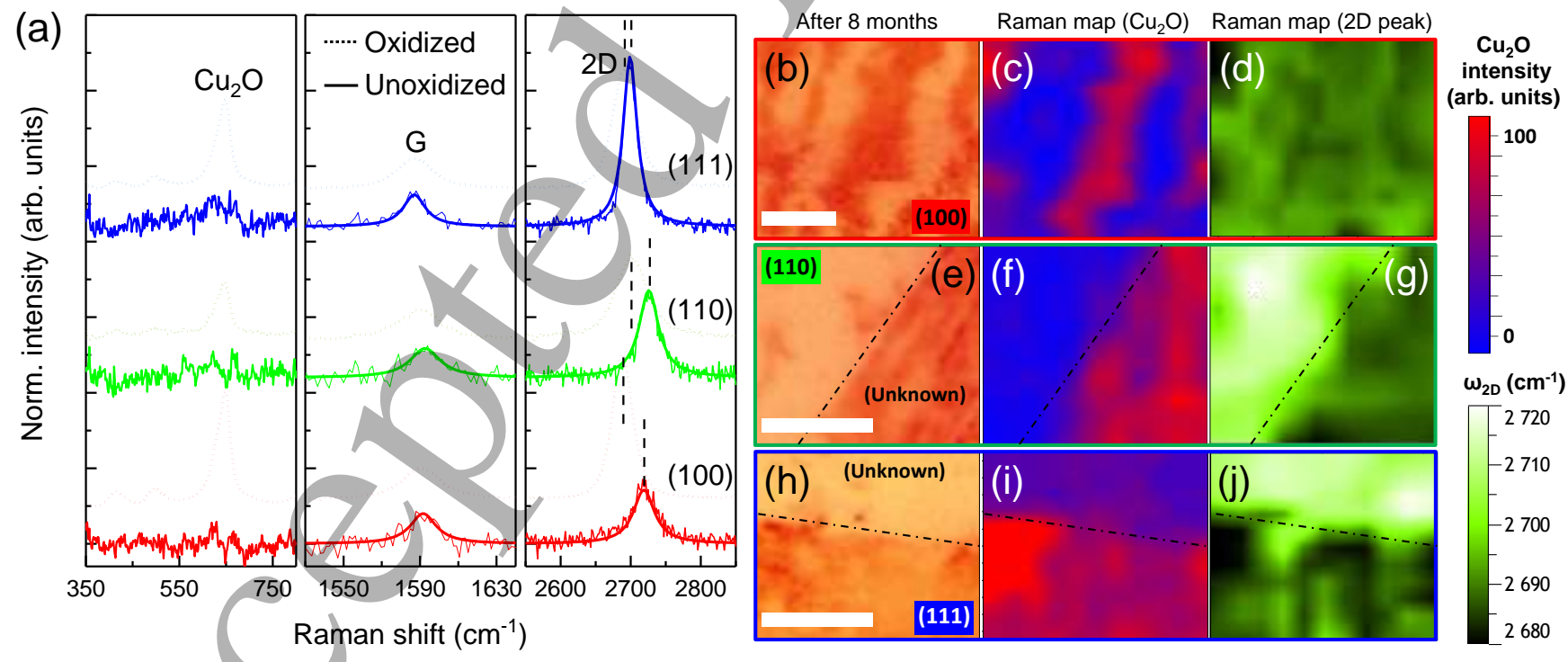

Figure 4. (a) Representative Raman spectra of the three studied grain orientations before (solid lines) and after (dotted lines) being oxidized in ambient conditions for eight months. Each spectrum has been background corrected and Lorentzian fits are shown in the unoxidized $G$ and $2 \mathrm{D}$ peaks to facilitate comparison. (b-j) Optical micrographs of different grains (including those

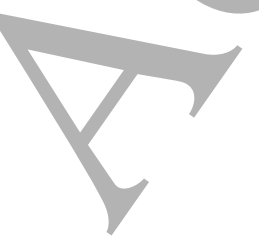


of the three studied orientations), and their corresponding Raman maps of the $\mathrm{Cu}_{2} \mathrm{O}$ peak intensity and the 2D peak frequency $\left(\omega_{2 D}\right)$. Dashed lines highlight the grain boundaries. The scale bar is $5 \mu \mathrm{m}$ in all images.
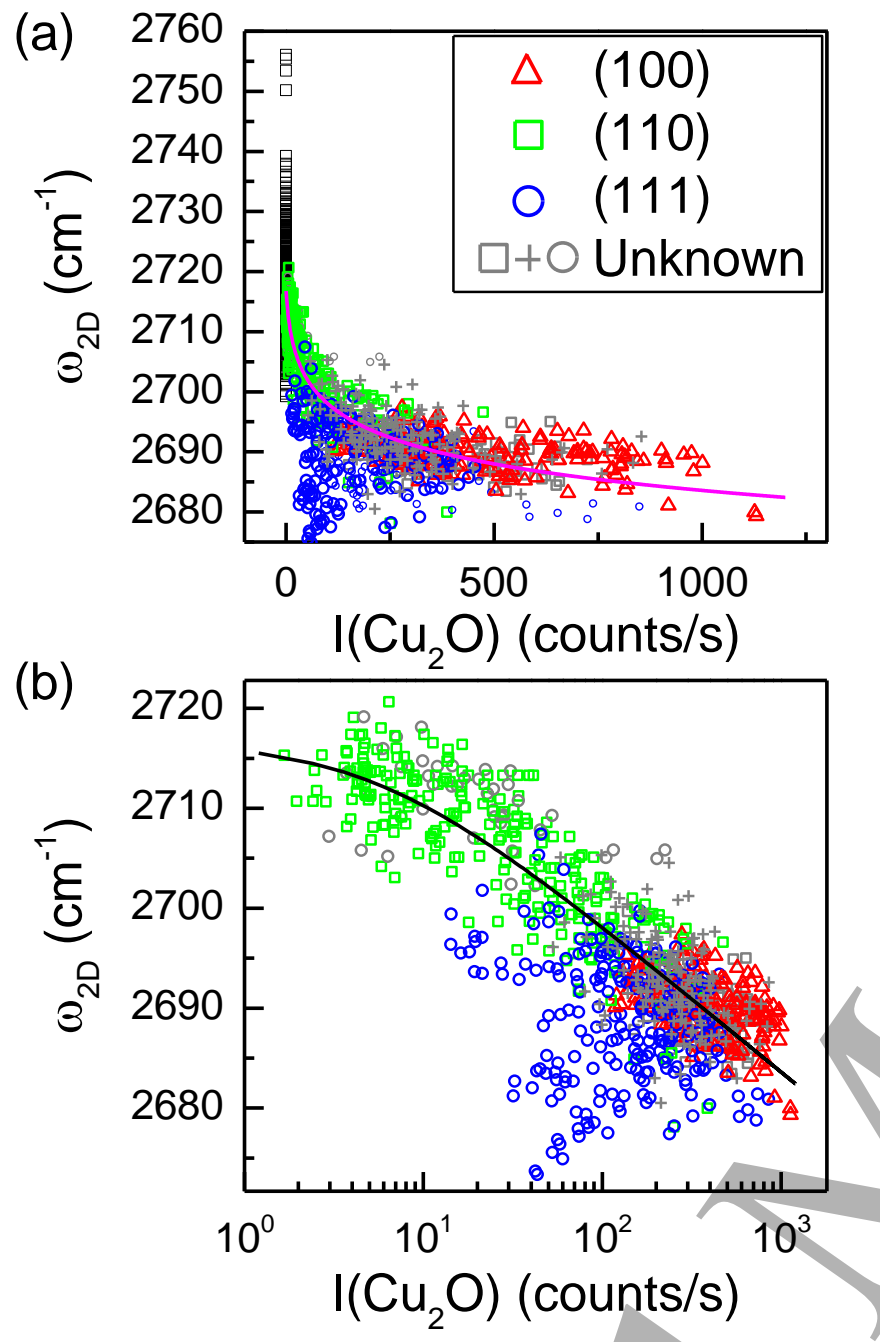

Figure 5. 2D Raman peak frequency vs. the intensity of the $\mathrm{Cu}_{2} \mathrm{O}$ Raman peak recorded at different points of the three grain orientations. Black squares represent the $2 \mathrm{D}$ peak distribution of an as grown (non-oxidized) sample. The logarithmic dependence is highlighted in (b). Solid lines are the fit to the phenomenological equation $y=A-B \ln (x+C)$.

Shifts in both the $G$ and 2D Raman peaks of graphene are usually produced by a combination of strain and doping due to the interaction with the substrate. Each contribution can be separated by plotting the $2 \mathrm{D}$ peak position against the $\mathrm{G}$ peak position in a 2D-G diagram as those shown in figure 6 [38]. Strain shifts proportionally both peaks in the same direction and therefore the points in the diagram move along a single line with a slope $\Delta \omega_{2 D} / \Delta \omega_{G}$ of 2.2 , as determined by Lee et al. [38]. The strain line crosses the point of freestanding graphene, shown by the purple dot in figure 6 , which we have estimated to be $\omega_{G} \approx 1581 \mathrm{~cm}^{-1}$ and $\omega_{2 D} \approx 2690 \mathrm{~cm}^{-1}$. These values are in agreement with previous measurements reported in the literature, either directly obtained at $488 \mathrm{~nm}$ excitation wavelength [15] or by extrapolation from values measured at different excitation wavelengths [29, 30, 38-42], assuming a variation in $\omega_{2 D}$ with excitation energy of $\Delta \omega_{2 D} / \Delta E_{\text {exc. }}=100$ $\mathrm{cm}^{-1} \mathrm{eV}^{-1}[5,28,30,43,44]$. The location of the data with respect to the point of freestanding graphene determines whether the strain is compressive (larger $\omega_{G}$ and $\omega_{2 D}$ ) or tensile (smaller $\omega_{G}$ and $\omega_{2 D}$ ). Doping, on the other hand, always shifts the position of the $G$ peak towards higher frequencies, while having a small effect on the $2 \mathrm{D}$ peak: $\mathrm{p}$ doping slightly shifts the 2D peak, with a slope of $\sim 0.75$ on the 2D-G diagram, and n-doping leaves its position almost unaffected for low carrier concentrations. Therefore, the net contribution of doping is to displace the points away from the strain line to higher $\omega_{G}$ values. The $2 \mathrm{D}-\mathrm{G}$ diagram is thus divided in two regions by the strain line; the upper-left region, which is inaccessible (forbidden) by any combination of strain and doping, and the lower-right region where all the data points should lie, as indicated in figure 6 .

The 2D-G diagram obtained on six randomly oriented grains of the as grown (unoxidized) sample in shown in figure 6(a). The unoxidized sample presents a bimodal behavior: for rippled grains (blue symbols), which typically (but not exclusively) correspond to (111) and (100) orientations, the points are distributed around the strain line, revealing a negligible doping level, further confirmed by an average FWHM of the G peak of $15 \mathrm{~cm}^{-1}$ (see figure S2) [45-47], and an inhomogeneous compressive strain, in agreement with previous reports [5, 37, 48]. It is worth noting that despite some previous works have determined certain degree of doping on graphene on copper samples, careful angle resolved photoelectron spectroscopy (ARPES) measurements performed by Marsden et al. revealed no significant doping unless samples were annealed above $200{ }^{\circ} \mathrm{C}$ in ultra-high vacuum conditions [48]. Interestingly, for some grains, usually presenting smoother surfaces (green symbols), the points are shifted towards the forbidden region. This shift into the forbidden region is, in average, $\left\langle\Delta \omega_{2 D}\right\rangle \sim 10.3 \mathrm{~cm}^{-1}$ along the $\omega_{2 D}$ axis, larger than the data point dispersion of any of the studied grains $\left(\delta \omega_{2 D} \sim 6 \mathrm{~cm}^{-1}\right.$, marked by the red shadowed area in figure 6(a)), and thus cannot be explained as a statistical fluctuation. The forbidden region is only accessible at a very low doping level, where graphene $G$ peak is predicted to anomalously shift towards lower Raman frequencies [4547]; however the expected shift $\left(\sim 1 \mathrm{~cm}^{-1}\right)$ is significantly smaller than observed here. 


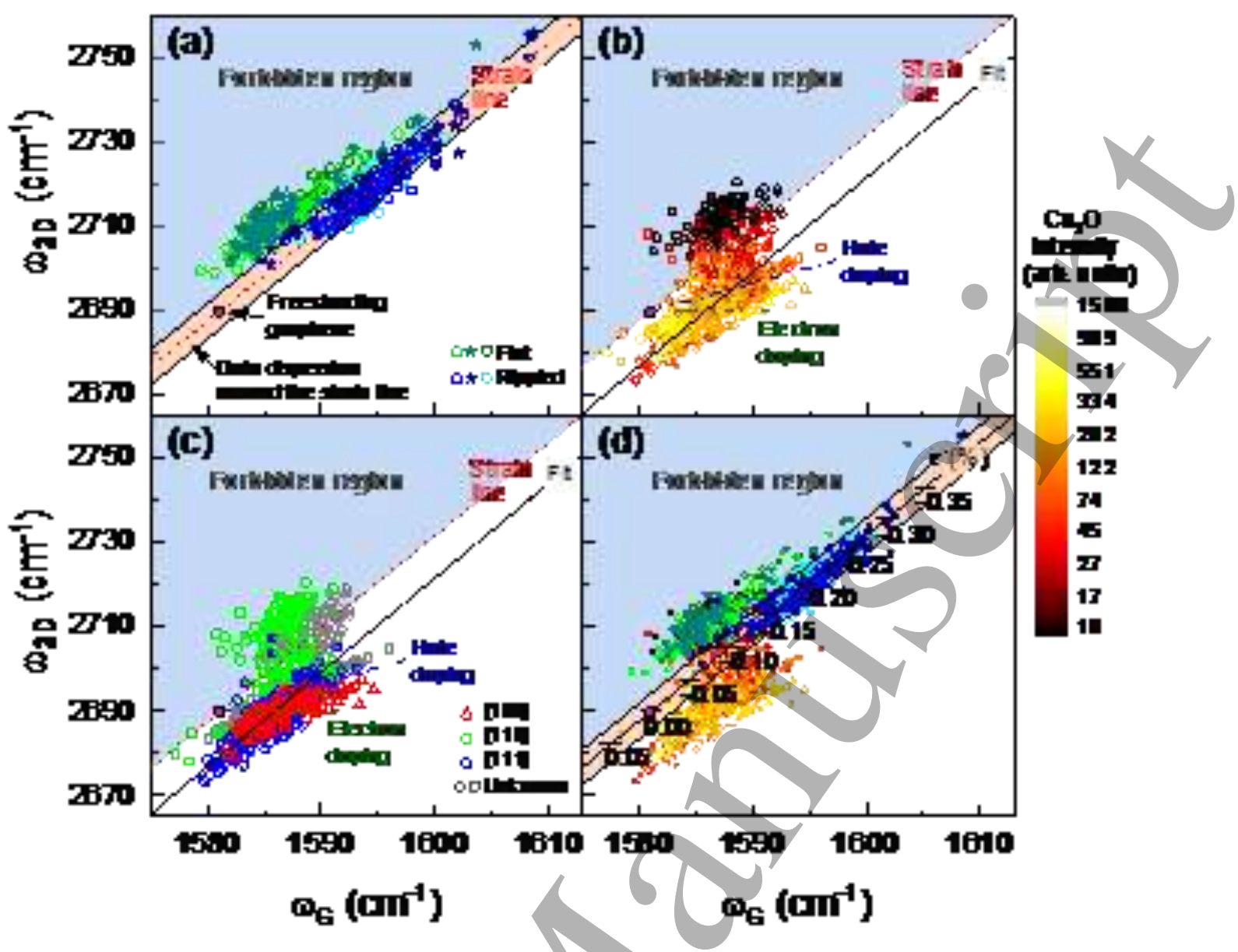

Figure 6. $2 \mathrm{D}-\mathrm{G}$ diagrams of two different $\mathrm{Gr} / \mathrm{Cu}$ foils. (a) As grown sámple with non-oxidized substrate. Different colors represent different grains. (b and c) Equivalent sample measured for a period of 8 months after being grown, showing an oxidized $\mathrm{Cu}$ substrate. Color code in (b) indicates the intensity of the $\mathrm{Cu}_{2} \mathrm{O}$ peak at each measured position, while the corresponding crystalline orientation is represented in (c). (d) Combination of data from both samples and their biaxial strain as estimated from their relative position [38] to the freestanding graphene (purple dot) along the strain line.

The observed compressive strain is a consequence of the difference in sign of the thermal expansion coefficient of graphene and the underlying $\mathrm{Cu}$, and usually leads to the formation of graphene wrinkles as those shown in figure 3(b). This strain can be either uniaxial or biaxial. The symmetry breakdown produced under uniaxial strain splits both the $G$ and $2 \mathrm{D}$ peaks into two components, $\mathrm{G}^{+}, \mathrm{G}^{-}$and $2 \mathrm{D}^{+}, 2 \mathrm{D}^{-}[39$, 40], while biaxial strain preseryes the symmetry of graphene lattice and does not change the line shape of the peaks [41]. The shift of the $G$ peak due to strain effects has been estimated to be $\Delta \omega_{G} / \Delta \varepsilon \sim-23.5 \mathrm{~cm}^{-1} / \%$ for uniaxial strain (averaged for $\mathrm{G}^{+}$and $\mathrm{G}^{-}$split peaks) $[39,40]$, and $\Delta \omega_{\mathrm{G}} / \Delta \varepsilon^{2} \sim-69.1 \mathrm{~cm}^{-1} / \%$ for biaxial strain $[38,41,49]$, so a compressive strain as large as $\varepsilon=-1.2 \%$ would be obtained if uniaxial strain is considered; enough to produce an appreciable splitting of the $G$ peak, regardless of the orientation of graphene with respect to the strain direction. Since all observed peaks can be fitted using just one component, a biaxial compressive strain must therefore be assumed, as expected for any strain produced by a difference in thermal expansion between graphene and copper (see figure 6(d) and Supporting Information S3 for further details).

Figure 6(b) shows the 2D-G diagram of a $\mathrm{Gr} / \mathrm{Cu}$ foil several months after being grown (between 4 and 8 months). The color code indicates the relative thickness of the $\mathrm{Cu}_{2} \mathrm{O}$ layer (oxidation degree) as determined by the intensity of its Raman peak at $640 \mathrm{~cm}^{-1}$ (black, not oxidized; red, slightly oxidized; yellow, very oxidized). As the sample starts getting oxidized the data points in the diagram shift down the strain line, indicating a relaxation of the compressive strain. At relatively low oxidation degrees (see also figure 5(a)), the data points jump away the strain line and continue moving downwards along a parallel line, depicted by the linear fit shown in the figure, indicating the presence of certain degree of doping. The fact that the data points keep moving parallel to the strain line points out to a constant doping level, which is produced by the interaction with the underlying $\mathrm{Cu}_{2} \mathrm{O}$, independently of its thickness. This is in contrast to the observations made by

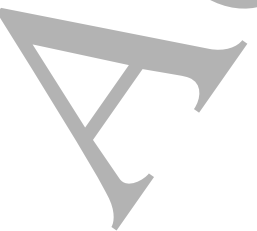


Yin et al. [14] who described an electrical decoupling of graphene upon oxidation, leading to undoped graphene on $\mathrm{Cu}_{2} \mathrm{O}$. Doping is reported to decrease the FWHM of the $\mathrm{G}$ peaks [46, 47, 50, 51], however, despite the significant doping level induced by the $\mathrm{Cu}_{2} \mathrm{O}$ layer, both the $\mathrm{G}$ and $2 \mathrm{D}$ peaks are broadened up to $\sim 25 \mathrm{~cm}^{-1}$ and $\sim 46 \mathrm{~cm}^{-1}$ respectively (see figure S3). This broadening, affecting both peaks, is most likely related to an increase in the inhomogeneity of the graphene strain/doping state (with a characteristic size below that of the laser spot) induced by an increased surface roughness upon $\mathrm{Cu}_{2} \mathrm{O}$ growth, as reported by Yin et al. [14]. The surface roughening would be responsible also for the compressive strain release of graphene: $\mathrm{Cu}_{2} \mathrm{O}$ Raman peaks do not shift as the oxide layer grows thicker, indicating a negligible change in its lattice parameter; therefore the direct coupling of graphene with the larger lattice parameter of $\mathrm{Cu}_{2} \mathrm{O}$ cannot account for its progressive strain relaxation, in agreement with the observed mechanical decoupling upon $\mathrm{Cu}$ oxidation $[16,17]$.

As in the case of the as grown graphene (non-oxidized substrate), a dense bunch of points remains anomalously located into the forbidden region at a statistically significant distance from the strain line. These data points were taken from areas (or grain areas) which had not undergone any significant oxidation and correspond precisely to (110) grains or other grains with smooth surfaces, as shown in figure $6(\mathrm{c})$ These grains are precisely those that are more resilient to oxidation, and present unoxidized areas even a year after the sample was grown, as discussed before. Interestingly, once $\mathrm{Cu}_{2} \mathrm{O}$ nucleates and grow on those particular grains the observed anomalous shift is completely removed in the areas directly above the oxide patches, but it is preserved on the yet unoxidized areas, as shown in figure S4. Comparison between figures 6(b) and (c) shows how data points obtained in oxidized (110) grain areas lie in the same region of the diagram as other similarly oxidized (100) and (111) grains. This strongly suggests that the shift is produced by the interaction between graphene and the $\mathrm{Cu}$ substrate at those particular grains. The trend followed by the data points after oxidation seems very weakly dependent on the grain orientation, in agreement with data from figure 5. A summary of all data obtained for both unoxidized and oxidized samples is presented in figure $6(\mathrm{~d})$, showing the evolution of graphene $2 \mathrm{D} / \mathrm{G}$ peaks with oxidation degree, as well as the estimated biaxial strain.

The origin of the observed anomalous shift is still unclear, but this phenomenon is not unique to our work. Indeed, some previous reports of graphene on $\mathrm{Cu}$ have shown anomalously large 2D Raman peak frequencies when compared to the corresponding $G$ peak position [5, 17]. Nevertheless, this phenomenon has been generally overlooked, attributed to inaccuracies, or directly ignored. Whelan et al. [17] used the disappearance of a high frequency $2 \mathrm{D}^{+}$peak and the appearance of a different low frequency $2 \mathrm{D}^{-}$peak as indicator for the mechanical decoupling of graphene during the oxidation of the $\mathrm{Cu}$ substrate; however, they did not discuss the physical origin of these two components. Frank et al. [5] estimated a higher than expected unstrained 2D peak frequency, $\omega_{2 D \text {,free }}$, for as-grown graphene samples on $\mathrm{Cu}$ single crystals by extrapolating their data to the unstrained $\omega_{G, \text { free }}$ value. They partially attributed this discrepancy to a reduced dependence of the $2 \mathrm{D}$ band with excitation energy (due to a slight substrate-induced doping), which would lead to higher $\omega_{2 D \text { free }}$ when extrapolating their values (obtained at $1.96 \mathrm{eV}$ ) to the larger excitation energies usually employed in the literature (typically $2.41 \mathrm{eV}$, i.e. $514 \mathrm{~nm}$ ). However, the excitation energy used in our work is $2.54 \mathrm{eV}$, and therefore a smaller dispersion of the 2D band would imply an extrapolated lower $\omega_{2 D \text { free }}$ value, contrary to our observations (see figure S5).

Twisted (turbostratic) graphene bilayers have been shown to present similar shifts of the $2 \mathrm{D}$ peak position, depending on their relative rotation angle [52-54]. The origin of this shift is believed to be related either to the renormalization of the Fermi velocity of graphene, $v_{F}$, or to the formation of van Hove singularities, although there is some controversy on this issue [53, 55-57]. These systems are characterized by single component 2D peaks, contrary to the usual four components of Bernal ( $A B$ stacking) bilayers, and their $G$ to 2D peak intensity depends on the rotation angle, making it difficult to distinguish them from monolayer graphene just by means of Raman spectroscopy. Nonetheless, we do not see any evidence of bilayer graphene in our samples, such as the reported change in reflectance (optical microscopy) and electron yield (SEM) compared to monolayer regions [58]. Besides, if the presence of twisted bilayers was responsible for the observed anomalous shift of the 2D peak, it would imply that they totally cover particular grains with specific orientations and surface morphologies (smooth surfaces parallel to (110) planes), without spreading to adjacent grains. However, graphene is known to cross grain boundaries during its growth, spreading to adjacent grains, and bilayers usually appear as isolated spots (micron size) when grown by CVD on $\mathrm{Cu}$ [59]. Moreover, the fact that the anomalous shift is removed when the samples get oxidized without changing the single component nature of the Raman peaks strongly points to some sort of interaction between graphene and the $\mathrm{Cu}$ substrate and not between two rotated graphene layers (see figure S4). This different (less coupled) graphene-substrate interaction at (110) grains could also explain the smoothness and oxidation resistance of these surfaces, as discussed above.

The lack of doping observed in our as grown $\mathrm{Gr} / \mathrm{Cu}$ samples agrees with a weak electronic coupling for the different surfaces, however, the anomalous shift of the $2 \mathrm{D} / \mathrm{G}$ peaks measured on (110) grains points to the presence of a non-negligible interaction for those surfaces. A way this 
interaction may produce the observed behavior might be through a decrease in $v_{F}$ of graphene, similar to the case of twisted graphene bilayers. For instance, it has been predicted that the presence of a uniform electric field could modify the $v_{F}$ of graphene [60]. This decrease would in turn increase the Raman frequency of the 2D peak without affecting the position of the $G$ peak. However, a reduction of the Fermi velocity would produce a smaller shift of the $2 \mathrm{D}$ peak for lower excitation energies [53], while either a similar or larger shift is observed when an excitation energy of $1.88 \mathrm{eV}$ is used (see figures S6 and S7).

A different explanation would lie in the recently discovered bimodal shape of the 2D band for freestanding graphene. Luo et al. [30] reported an asymmetric 2D band for freestanding graphene which could be fitted to two Lorentzian peaks: the first one, at lower frequency, $\omega_{2 D^{-}}$, accounting for most of the intensity of the 2D band, and a second one at higher frequency, $\omega_{2 D}{ }^{+}$, giving rise to the high frequency tail. This asymmetric shape was mentioned in previous works [29], however its origin was not explored until very recently. Luo et al. [30] related the presence of both components to the two possible scattering paths responsible for the formation of the 2D band, the so called inner and outer processes. Due to the trigonal warping effect, the scattering process along the $K \Gamma$ direction (outer process) is more energetic compared to the $K M$ direction (inner process) (see figure S6). Despite the wavevector nesting is larger for the outer process, suggesting a higher intensity for the $\omega_{2 D}{ }^{+}$component [31], theoretical calculations including quantum interference and the $q$ dependent scattering matrix elements of the phonon intensity showed that the inner process $\omega_{2 D}{ }^{-}$is the dominant one [61]. The symmetric shape of the 2D band is usually recovered on supported graphene samples. Berciaud et al. [32] related the broadening and symmetry recovery of the $2 \mathrm{D}$ band to the effect of doping as both components tend to merge when the doping level is increased. The reported frequency splitting of the $\omega_{2 D^{-}}$and $\omega_{2 D^{+}}$components $\left(\sim 15 \mathrm{~cm}^{-1}\right)$ is very similar to the average anomalous shift observed in this work $\left(\sim 10.3 \mathrm{~cm}^{-1}\right)$. Figure 7 shows the Raman frequency of the $\omega_{2 D}{ }^{-}$and $\omega_{2 D^{+}}$ components reported by several works for freestanding graphene as a function of excitation energy. The values obtained in our work by extrapolating both the normal (pink, crossed circle) and anomalous (blue, crossed diamond) data to the unstrained graphene $\omega_{G} \sim 1581 \mathrm{~cm}^{-1}$ are shown for comparison. The same has been done with data from the work of Frank et al. [5], where an anomalously high frequency of the $2 \mathrm{D}$ band was observed for graphene on $\mathrm{Cu}$ single crystals (blue diamond with a dot in the center).

Both our values from the anomalous region, as well as those reported by Frank et al., fit quite well within the trend of the $\omega_{2 D^{+}}$component, pointing to a dominant outer, $\omega_{2 D^{+}}$, process as the origin of the anomalously high frequency of the $2 \mathrm{D}$ band. This is in contrast to the usual higher intensity of the inner process. Luo et al. [30] and Berciaud et al. [32] observed an enhanced (decreased) intensity of the outer (inner) process with decreasing excitation energy; however, for the excitation energy used in our work the inner process $\left(\omega_{2 D^{-}}\right)$should dominate the spectrum. The contribution from the outer process usually gets negligible or degenerates to that of the inner process at larger graphene substrate coupling, which again points to a lower graphene-substrate coupling at (110) grains. Charge doping has been reported to switch the intensity from the inner to the outer component (at very low doping levels) in graphene layers embedded in h-BN [31], however, doping also shifts the outer component to lower frequencies until both peaks are merged, so it cannot explain our observations. Therefore, further theoretical work would be necessary to elucidate the exact origin of the observed suppression (or enhancement) of the inner (outer) process on the graphene-Cu system.

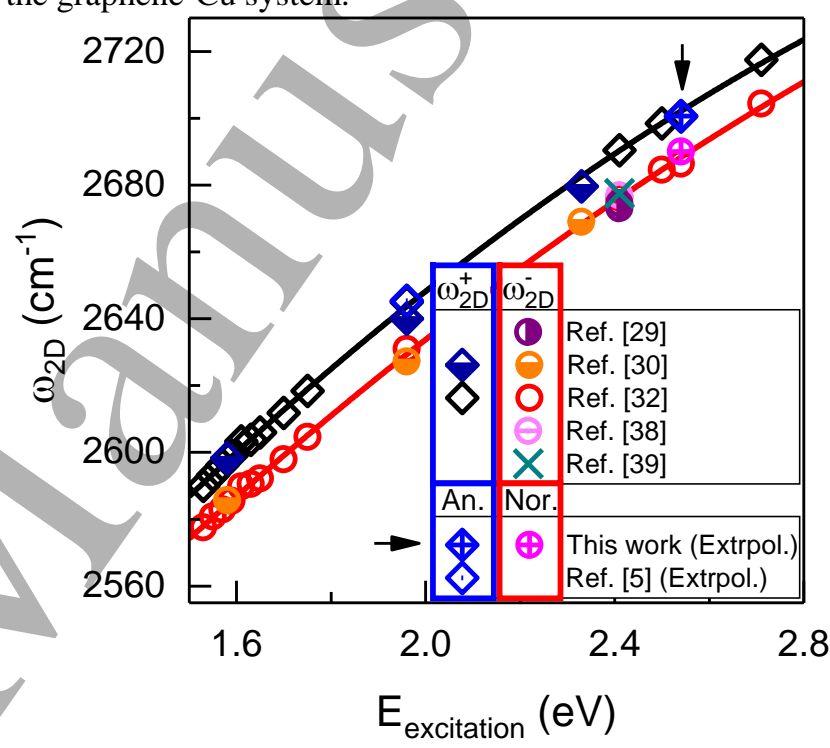

Figure 7. Raman frequency of the two components of the 2D peak $\left(\omega_{2 D^{-}}\right.$and $\left.\omega_{2 D^{+}}\right)$as a function of excitation energy as reported in several works for freestanding graphene. Both the data from reference 32 as well as their corresponding second order polynomial fits (solid lines) have been shifted upwards by $6 \mathrm{~cm}^{-1}$ to match the data reported by the rest of the references. Points from reference 5 as well as those obtained in this work have been extrapolated to the point of freestanding graphene $\left(\omega_{G} \sim 1581 \mathrm{~cm}^{-1}\right)$, assuming a strain shift of $\Delta \omega_{2 D} / \Delta \omega_{G}=2.2$ and negligible doping. Please note that both in our work and in [5] all $2 \mathrm{D}$ peaks were fitted to a single Lorentzian profile, so each point here correspond to the extrapolation of data from either the normal (Nor.) or the anomalous (An.) regions.

\section{Conclusion}

In summary, the interaction between graphene and its $\mathrm{Cu}$ substrate plays an important role not only in the morphology 
and oxidation of the $\mathrm{Cu}$ substrate, but also in the Raman scattering processes of graphene. The surface morphology and oxidation rates of graphene covered $\mathrm{Cu}$ strongly depends on their coupling at different $\mathrm{Cu}$ grain orientations. For (100) and (111) grains graphene leads to the formation of ripples on the $\mathrm{Cu}$ surface and increases its long term oxidation as compared to the uncovered case. Conversely, (110) surfaces remain unaltered after graphene growth and present almost pristine surfaces for extremely long periods of time. These differences are attributed to a reduced graphene- $\mathrm{Cu}$ coupling at (110) surfaces which, on the one hand, permits the proper diffusion of $\mathrm{Cu}$ ions below graphene, avoiding the step bunching process that leads to the formation of ripples and, on the other hand, hinders the charge exchange between graphene and $\mathrm{Cu}$ surface, necessary for the oxidation of the substrate. Therefore, it is shown that CVD graphene can actually work as protective layer (either for $\mathrm{Cu}$ or other materials) provided it is properly decoupled from the substrate. Considering the new advances performed in the detachment of CVD graphene from its growth substrate these results can have important implications for the industry beyond its use as anticorrosion coating of different metallic substrates. In particular, graphene could also be used in all-2D-based devices, both as active region (or electrode) and as protective layer of other $2 \mathrm{D}$ materials such as phosphorene or transition metal dichalcogenides, which tend to degrade under ambient conditions. This would span the range of functionality of protective 2D coatings, which are currently represented mainly by the highly insulating hexagonal boron nitride $(\mathrm{hN})$.

The weak coupling at (110) surfaces is correlated to an anomalous Raman shift of the 2D peak which cannot be explained in terms of the conventional strain-doping mechanisms. Other effects, such as the presence of uniaxial strain, the renormalization of the Fermi velocity or a reduced dispersion relationship have also been ruled out as the origin of the shift. It is thus concluded that the most likely explanation for the phenomenon is an enhanced outer Raman emission $\left(2 \mathrm{D}^{+}\right)$, in contrast to the usually stronger inner process $\left(2 \mathrm{D}^{-}\right)$. This enhancement is believed to be a consequence of the reduced graphene- $\mathrm{Cu}$ coupling. Our results suggest that the process leading to the bimodal line shape of the $2 \mathrm{D}$ band is more complex than previously expected, as already pointed by other authors, and reveal the necessity of performing further theoretical studies in order to elucidate its origin and evolution with the graphene-substrate interaction. Given the wide usage of Raman spectroscopy not only in the academy, but also in the industry of graphene, understanding these processes is of paramount importance for the proper characterization of supported graphene.

\section{Acknowledgements}

Funding by the Spanish Ministerio de Economía y Competitividad (MINECO) under Project MAT2015-65356-
C3-1-R and Comunidad de Madrid Excellence Network under Project S2013/MIT-2740 is acknowledged. L.A-F. and S. C. acknowledge FPI grants (BES-2013-062759 and BES-2016076440 respectively) from MINECO.

\section{References}

[1] Wofford J M, Nie S, McCarty K F, Bartelt N C and Dubon O D 2010 Nano Lett. 104890

[2] Zhang J, Wang Z, Niu T, Wang S, Li Z and Chen W 2014 Sci. Rep. 44431

[3] Wood J D, Schmucker S W, Lyons A S, Pop E and Lyding J W 2011 Nano Lett. 114547

[4] Jacobberger R M and Arnold M S 2013 Chem. Mater. 25871

[5] Frank O, Vejpravova J, Holy V, Kavan L and Kalbac M 2014 Carbon 68440

[6] Luo B, Whelan P R, Shivayogimath A, Mackenzie D M A, Bøggild P and Booth T. J 2016 Chem. Mater. 283789

[7] Zhou F, Li Z, Shenoy G J, Li L and Liu H 2013 ACS Nano 7 6939

[8] Schriver M, Regan W, Gannett W J, Zaniewski A M, Crommie M F and Zettl A 2013 ACS Nano 75763

[9] Galbiati M, Stoot A C, Mackenzie D M A, Bøggild P and Camilli L 2017 Sci. Rep. 739770

[10] Álvarez-Fraga L, Rubio-Zuazo J, Jiménez-Villacorta F, Climent-Pascual E, Ramírez-Jiménez R, Prieto $\mathrm{C}$ and de Andrés A 2017 Chem. Mater. 293257

[11] Gao J, Li B, Tan J, Chow P, Lu T-M and Koratkar N, 2016 ACS Nano 102628

[12] Wood J D, Wells S A, Jariwala D, Chen K-S, Cho E K, Sangwan V K, Liu X, Lauhon L J, Marks T J and Hersam M C 2014 Nano Lett. 146964

[13] Gaoxue W, William J S, Ravindra P and Shashi P K 2016 2D Mater. 3025011

[14] Yin X, Li Y, Ke F, Lin C, Zhao H, Gan L, Luo Z, Zhao R, Heinz T F and Hu Z 2014 Nano Res. 71613

[15] Ramírez-Jiménez R, Alvarez-Fraga L, JiménezVillacorta F, Climent-Pascual E, Prieto C and de Andrés A 2016 Carbon 105556

[16] Wu R, Gan L, Ou X, Zhang Q and Luo Z 2016 Carbon 98 138

[17] Whelan P R, Jessen B S, Wang R, Luo B, Stoot A C, Mackenzie D M A, Braeuninger-Weimer P, Jouvray A, Prager L, Camilli L, Hofmann S, Bøggild P and Booth T J 2017 Carbon 11775

[18] Cabrera N and Mott N 1949 Rep. Prog. Phys. 12163

[19] Young F W, Cathcart J V and Gwathmey A T 1956 Acta Metall. 4145

[20] Wilkins F J and Rideal E K 1930 Proc. R. Soc. London, Ser. A 128394

[21] Fromhold A T and Anderson M H 2004 Oxid. Met. 62 237

[22] Platzman I, Brener R, Haick H and Tannenbaum T 2008 J. Phys. Chem. C 1121101

[23] Lian X, Xiao P, Yang S-C, Liu R and Henkelman G 2016 J. Chem. Phys. 145044711

[24] Zhou G and Yang J C 2004 Surf. Sci. 559100

[25] Jeon B, Sankaranarayanan S K R S, van Duin A C T and Ramanathan S 2011 Philos. Mag. 914073 
[26] Hu J, Xu J, Zhao Y, Shi L, Li Q, Liu F, Ullah Z, Li W, Guo Y and Liu L 2017 Sci. Rep. 745358

[27] Ferrari A C and Basko D M 2013 Nat. Nano. 8235

[28] Malard L M, Pimenta M A, Dresselhaus G and Dresselhaus M S 2009 Phys. Rep. 47351

[29] Berciaud S, Ryu S, Brus L E and Heinz T F 2009 Nano Lett. 9346

[30] Luo Z, Cong C, Zhang J, Xiong Q and Yu T 2012 Appl. Phys. Lett. 100243107

[31] Wang X, Christopher J W and Swan A K, 2017 Sci. Rep. 713539

[32] Berciaud S, Li X, Htoon H, Brus L E, Doorn S K and Heinz T F 2013 Nano Lett. 133517

[33] Rodhin T N 1951 J. Am. Chem. Soc. 733143

[34] Kusano K F, Uchikosi M, Mimura K and Isshiki M 2014 Oxid. Met. 82181

[35] Hayashi K, Sato S and Yokoyama N 2013 Nanotechnology 24025603

[36] Wlasny I, Dabrowski P, Rogala M, Kowalczyk P J, Pasternak I, Strupinski W, Baranowski J M and Klusek Z 2013 Appl. Phys. Lett. 102111601

[37] Wilson N R, Marsden A J, Saghir M, Bromley C J, Schaub R, Costantini G, White T W, Partridge C, Barinov A, Dudin P, Sanchez A M, Mudd J J, Walker M and Bell G R 2013 Nano Res. 699

[38] Lee J E, Ahn G, Shim J, Lee Y S and Ryu S 2012 Nat. Commun. 31024

[39] Yoon D, Son Y-W and Cheong H 2011 Phys. Rev. Lett. 106155502

[40] Mohiuddin T M G, Lombardo A, Nair R R, Bonetti A, Savini G, Jalil R, Bonini N, Basko D M, Galiotis C, Marzari N, Novoselov K S, Geim A K and Ferrari A C 2009 Phys. Rev. B 79 205433

[41] Androulidakis C, Koukaras E N, Parthenios J, Kalosakas G, Papagelis K and Galiotis C 2015 Sci. Rep. 518219

[42] Filintoglou K, Papadopoulos N, Arvanitidis J, Christofilos D, Frank O, Kalbac M, Parthenios J, Kalosakas G, Galiotis C and Papagelis K 2013 Phys. Rev. B 88045418

[43] Vidano R P and Fischbach D B 1981 Solid State Commun. 39341

[44] Pócsik I, Hundhausen M, Koós M and Ley L 1998 J. Non-Cryst. Solids 227-230 1083

[45] Ferrari A C 2007 Solid State Commun. 14347

[46] Das A, Pisana S, Chakraborty B, Piscanec S, Saha S K, Waghmare U V, Novoselov K S, Krishnamurthy H R, Geim A K, Ferrari A C and Sood A K 2008 Nat. Nanotechnol. 3210

[47] Pisana S, Lazzeri M, Casiraghi C, Novoselov K S, Geim A K, Ferrari A C and Mauri F 2007 Nat. Mater. 6198

[48] Marsden A J, Asensio M-C, Avila J, Dudin P, Barinov A, Moras P, Sheverdyaeva P M, White T W, Maskery I, Costantini G, Wilson N R and Bell G R 2013 Phys. Status Solidi RRL 7 643

[49] Nicholl R J T, Lavrik N V, Vlassiouk I, Srijanto B R and Bolotin K I 2017 Phys. Rev. Lett. 118266101

[50] Yan J, Zhang Y, Kìm P and Pinczuk A 2007 Phys. Rev. Lett. 98166802

[51] Lazzeri M and Mauri F 2006 Phys. Rev. Lett. 97266407

[52] Ni Z, Wang Y, Yu T, You Y and Shen Z 2008 Phys. Rev. B 77235403
[53] Poncharal P, Ayari A, Michel T and Sauvajol J-L 2008 Phys. Rev. B $\mathbf{7 8} 113407$

[54] Kim K, Coh S, Tan L Z, Regan W, Yuk J M, Chatterjee E, Crommie M F, Cohen M L, Louie S G and Zettl A 2012 Phys. Rev. Lett. 108246103

[55] Lopes dos Santos J M B, Peres N M R and Castro Neto A H 2007 Phys. Rev. Lett. 99256802

[56] Razado-Colambo I, Avila J, Nys J-P, Chen C, Wallart X, Asensio M-C and Vignaud D 2016 Sci Rep. 627261

[57] Ni Z, Wang Y, Yu T, You Yand Shen Z 2009 Phys. Rev. B 79237401

[58] Havener R W, Zhuang H, Brown L, Hennig R G and Park J 2012 Nano Lett. 123162

[59] Mattevi C, Kim H and Chhowalla M 2011 J. Mater. Chem. 213324

[60] Díaz-Fernández A, Chico L, González J W and Domínguez-Adame F 2017 Sci. Rep. 78058

[61] May P, Lazzeri M, Venezuela P, Herziger F, Callsen G, Reparaz J S, Hoffmann A, Mauri F and Maultzsc J 2013 Phys. Rev. B 87075402 